\title{
Route choice dynamics after a link restoration
}

\author{
Carlos Carrion* $^{*} \quad$ David Levinson ${ }^{\dagger}$
}

August 1, 2012

\begin{abstract}
(1) studied the bridge choice behavior of commuters before and after a new bridge opened to the public. This bridge replaced the previously collapsed I- $35 \mathrm{~W}$ bridge in the metro area of Minneapolis-St. Paul. The original I-35W bridge collapsed on August 1st 2007, and the replacement bridge opened to the public on September 18th 2008. This study extends (1) by considering explicitly the day-to-day behavior of travelers, and by also considering the previously excluded subjects that are transitioning between bridge alternatives not including the I-35W bridge. The primary results indicate that the subjects react to day-to-day travel times on a specific route according to thresholds. These thresholds help discriminate whether a travel time is within an acceptable margin or not, and travelers may decide to abandon the chosen route depending on the frequency of travel times within acceptable margins. The secondary results indicate that subjects previous experience, and perception of the alternatives also influence their decision to abandon the chosen route.
\end{abstract}

Keywords: GPS, route choice, I-35W bridge, duration, hazard, survival.

Word Count: 5,946

Word Count incl. figures and tables: 7,446

\footnotetext{
*Corresponding Author, University of Minnesota, Department of Civil Engineering, carri149@umn.edu

${ }^{\dagger}$ RP Braun-CTS Chair of Transportation Engineering; Director of Network, Economics, and Urban Systems Research Group; University of Minnesota, Department of Civil Engineering, 500 Pillsbury Drive SE, Minneapolis, MN 55455 USA, dlevinson@umn.edu, http://nexus.umn.edu
} 


\section{Introduction}

The most basic wisdom of travel behavior is that travelers adapt to their circumstances according to their own knowledge inside the road network. This knowledge is the outcome of human-environment interaction related to the act of traveling. Travelers refine their movements in their surroundings through spatial, and temporal information acquisition. This information is connected to two guiding processes: navigation, and wayfinding. Navigation describes the actions required for unobstructed movement by linking locations of places, and trajectories between places. Wayfinding is about selecting routes connecting an origin-destination pair of interest to the traveler. In short, travelers learn about some of the places connected by the transportation system. Travelers learn about some of the distinct alternatives (i.e. mode, route) to navigate in the transportation system. Travelers learn about some of the states (e.g. peak hour congestion) of the transportation system at specific times during the day, week, month, and in some cases even year. Furthermore, travelers' may exercise any combination of several possible responses available to them that vary according to timing. In the short term, travelers potential responses include: rescheduling trips to earlier or later times; switching routes; canceling trips; and others. In the long term, travelers potential responses include: auto ownership; finding alternative location of activities; moving to a new residential and/or work location; and others. It is important to realize that travelers choose a proper bundle of potential responses depending on the characteristics of the travelers themselves, and of the physical environment. In essence, the travelers perceive the characteristics of the physical environment, and the travelers extract the relevant information according to their own criteria. This information is processed also according to their own criteria, and a bundle of possible responses is chosen. Lastly, this selection process is dynamic; it receives feedback (e.g. past experience) from the travelers' previous decisions $(2,3,4,5,6)$.

This study focuses on uncovering the dynamics of travel behavior, more specifically, the dynamics of bridge choice behavior after a large-scale disruption. It investigates the day-to-day behavior of commuters after the opening of the replacement bridge for the previously collapsed I-35W bridge in the Minneapolis-St. Paul metropolitan region. The original I-35W bridge collapsed on August 1st 2007, and the replacement bridge opened to the public on September 18th 2008. The primary objective of this study is to identify the factors that influence the day-to-day subjects' decision to stay or abandon their current chosen bridge, and in addition to determine the possible relationships between these factors. For this purpose, this study analyzes data collected of commuters recruited from a previous research effort (1). This data consists of Global Positioning System (GPS) points, and web-based surveys. This data was collected before and after the replacement bridge opened. The GPS data of the subjects contains geographical points between the last weeks of August 2008, and the first weeks of December 2008.

The study is organized as follows: literature review of the relevant research to the topic at hand; data (description, and methodology); econometric models (specification, and estimation); discussion and results; and conclusions.

\section{Literature Review}

Typically, transportation research in route choice behavior has focused on three categories: traveler's knowledge of alternative routes; decision processes of travelers; and the influence of attributes of the traveler-road network system in travelers' route preferences. The first consists of analyzing the criteria travelers adopt to include routes in their set of possible routes. The second focuses in the rules followed by the travelers to select their final decisions. The third examines the effect of attributes in travelers' route preferences (6). 
Most of the early research found travel time and travel distance as the main explanatory attributes for traveler's route preferences $(7,8,9,10,11,12)$. However, research has shown that route choice behavior is not entirely encapsulated by travel time and travel distance. Other factors are also linked to the explanation of this phenomenon. These other factors include but are not limited to: travel time variability/reliability $(13,14,15,16)$; travel cost $(13,14,15,16)$; aesthetics of scenery (17); traffic information $(17,18)$; and others (19).

Travel time reliability is closely linked to the unpredictable variations due to the uncertainty of travel time. This uncertainty has been divided in three elements by (20): variation between seasons and days of the week; variation by changes in travel conditions because of weather and crashes or incidents; and variations attributed to each travelers perception. (21) lists also the components of uncertainty as variations in the link flows and variations in the capacity. Therefore, the unpredictable variations trace their source at both the demand side (e.g. traveler's heterogeneous behavior) and supply side (e.g. traffic signal failure) of a transportation system. This implies that travelers must choose routes under an uncertain transportation network as they may not predict their exact travel time before departing from their origins to their destinations. Moreover, travel time reliability is considered interchangeable with travel time variability in the transportation research literature. Thus, travel time is seen as a probability distribution. This means that travel time is associated with two dimensions: frequency, and magnitude.

Most route choice studies in the transportation literature have focused on the estimation of the value of travel time reliability. This value refers to the marginal rate of substitution between travel cost, and increases in the reliability (i.e. reducing the variability) of travel time. The dominant method for the estimation of the value of travel time reliability is discrete choice analysis typically within the Random Utility framework (22, 23, 24). The estimation has mostly been done using stated preference data from hypothetical choice experiments. These choice experiments present scenarios with myriad of presentations to travelers. The main concern with these scenarios is whether the subjects understand the representations of travel time variability being presented to them. A secondary concern is whether subjects can perceive the situation on the hypothetical experiment as similar to actual experiences in the actual transportation network. There are few studies using revealed preference data because of few examples of experimental settings with significant travel time variation across at least two alternatives (e.g. high occupancy toll lanes); difficulties with measuring travel time data; costs associated with planning (e.g. methodology of experiment) and deployment (e.g. surveys, devices to measure travel time) of revealed preference studies; and others. Furthermore, the stated choice experiments are far more common than collected revealed preference observations for the measurement of values of travel time reliability. In addition, revealed preference studies are using measured travel time distributions as obtained from a device (e.g. loop detector, GPS device). Thus, the perception error of travelers with regards to travel time has been largely ignored.

Travel time reliability has been incorporated in route choice studies as different measures of variability of the travel time distribution. These measures are generally centered on two theoretical frameworks: CentralityDispersion (or Mean-Variance) proposed by (25); and Scheduling delays under uncertainty proposed by $(26,27)$. The first is based on the idea that the travel time unreliability (or variability) is concentrated in a statistical measure of the dispersion of the travel time distribution. The second assumes that travelers have a specified time of arrival, and any expected late arrivals or expected early arrivals incurs disutilities. These disutilities are asymmetric in contrast to the Centrality-Dispersion framework that assumes all disutilities (due to unreliability) are weighted equally. It should be noted that expected refers to the first statistical moment of schedule delays due to late arrivals or early arrivals over the travel time distribution. Readers may refer to (28) for an extensive review on the value of travel time reliability 


\subsection{Limitations and discussion}

In the transportation research literature, models that incorporate travel time reliability are static models, and Random Utility models (see $(22,23,24)$ for details about Random Utility Theory). The models are static, because they only consider trade-offs of statistical measures on travel time distributions of a set of alternatives (e.g. routes, bridges). The travel time distributions may be hypothetical (stated preference data) or day-to-day travel times (revealed preference data). In stated preference studies, the travel time distribution of each alternative is generated by the researchers. In addition, the researchers are familiar with the value of different statistical measures (e.g. mean, median), based on the previous theoretical frameworks of the travel time distribution of each alternative. Thus, subjects simply observe a set of attributes of each alternative that are abstractions of the concept of travel time reliability/variability, and of expected travel time. These abstractions depend on different presentations (e.g. numbers and/or visual aid), and in cases may include the travel time distributions of the alternatives such as in the case of histograms in the stated choice questions. On the other hand, revealed preference studies produce travel time distributions for an alternative by aggregating travel times of different days of the alternative. Statistical measures are computed on these day-to-day travel times, and it is assumed that these measures represent the experience of the subjects with regards to the travel time reliability/variability, and the expected travel time for each alternative. In essence, the dynamic behavior of the travelers have been neglected to favor an assumption that subjects, at the aggregate level, settle for a particular alternative. However, this assumption neglects that subjects may be exercising their decisions at a day to day level. In addition, the subjects may only consider a subset of the day to day travel times for each alternative, and that this subset of day to day travel times is updated every few days. In other words, subjects may consider adding to the set day-to-day travel times according to their perception, and the subjects may discard day-to-day travel times according to their limited memory. Furthermore, the models are Random Utility models, and thus they are bound by the assumption that travelers as utility-maximizers know the expected travel time, and the travel time variability of each alternative. Travelers are informed with regards to the attributes related to travel time (i.e. means, standard deviation) for each alternative. This is a fundamental assumption in Random Utility models based on the theory of rational choice presented in (29). It is possible to include only the travel times known for certain alternatives, but this implicitly assumes that the travel times of the alternatives, that excludes them, are of the highly unlikely value of zero. This is also a questionable assumption. In fact, questions arise about whether a subject truly knows of the travel time of his other alternatives, or a subject only knows the travel time obtained from past accumulated experience. In addition, questions arise about the modus operandi of a subject to choose whether to stay or abandon the current chosen alternative based on its day to day travel times. In light of this discussion, researchers must ask whether stated preference studies are presenting a decision-making situation that is realistic, and whether revealed preference studies with travel time distributions obtained by aggregating day to day travel times are simplistic. Lastly, it may be argued that the few studies with dynamic Random Utility models (a revealed preference example is (16)) are not able to overcome discussed issues satisfactorily. Stated preference studies with multiple choice situations are considered more likely to be cases of unobserved heterogeneity rather than dynamics. Revealed preference studies (for example (16)) are able to include the dynamics by calculating the statistical measures on day-to-day travel time distributions that are updated during each choice situation of a decision-maker. This update is accomplished by including the new travel times for each choice situation. Unfortunately, it requires significant data collection by the researchers of trips of subjects on each alternative, and the assumption that subjects are informed, and always remember all past travel times are still present. It should be noted that these concerns are similar to those put forward in a more general setting by (30) (i.e. bounded rationality). 


\section{Data}

The data used was collected by a previous research effort described in (1). The main objective of this study is to understand the travel behavior of commuters after the replacement bridge for the previously collapsed I-35W bridge opened to the public (September 18th 2008) in the Minneapolis-St. Paul region. The data consists of GPS observations, and web-based surveys collected before, and after the replacement bridge opened. The data is further described subsequently.

\subsection{Recruitment}

Subjects were recruited through announcements posted in different media including: Craigslist.org, and CityPages.com; the free local weekly newspaper City Pages; flyers at grocery stores; flyers at city libraries, postcards handed out in downtown parking ramps; flyers placed in downtown parking ramps; and emails to more than 7000 University of Minnesota staff (students and faculty were excluded). More than 900 subjects responded, and consequently they were randomly selected among those the following requirements:

1. Age between 25-65,

2. Legal driver,

3. Full-time job and follow a "regular" work schedule

4. Travel by driving alone

5. Likelihood of being affected by the reopening of the new I-35W Mississippi River bridge.

Potential subjects (randomly selected from the respondents' pool) were instrumented with GPS devices by two data collection efforts. The first was headed by Dr. Randall Guensler at the Georgia Institute of Technology and the subcontractor Vehicle Monitoring Technologies (VMTINC). Also, a local subcontractor (MachONE) was employed to instrument the subjects' vehicles with GPS devices two weeks before the new I-35W bridge reopened. These GPS devices recorded the coordinates of the instrumented vehicle at every second between engine-on and engine-off events. The coordinates log collected by the GPS was transmitted to the server in real time through wireless communication. The subjects remained instrumented for 13 weeks without following any instructions with the exception of filling periodic surveys. The second was headed by the authors and others affiliated with the University of Minnesota, Twin Cities Campus. The subjects were instrumented with logging-type GPS devices (QSTARZ BT-Q1000p GPS Travel Recorder powered by DC output from in-vehicle cigarette lighter) also approximately two weeks before the replacement I$35 \mathrm{~W}$ bridge opened to the public. These GPS devices recorded the position of the instrumented vehicle at a frequency of 25 meters per location point registered between engine-on and engine-off events. These subjects remained instrumented for 8 weeks, during this time period the subjects followed their usual commute pattern without any instruction from the researchers. In addition, at the end of the study period (i.e. 8 weeks or 13 weeks depending on the data collection effort), subjects completed a comprehensive final web-based survey to evaluate the driving experience on routes using different bridges choices, provide socio-demographic information (see Section 3.3), and also answer some questions regarding route preferences.

A total of approximately 143 (46 by VMTINC, and 97 by University of Minnesota) subjects had usable (complete day-to-day GPS information) data required for this analysis. For this study, only 65 subjects (26 from VMTINC, and 39 from University of Minnesota) had the required data according to the subsequent Section 3.2. 


\subsection{Methodology}

The data analysis process can be divided in three phases:

1. Identification of morning commute trips per subject from GPS data on the bridges of interest (see Figure 1);

2. Information extraction (e.g. travel time) of commute trips per subject from GPS and survey data;

3. Specification and estimation of econometric models using the extracted information from GPS and from survey data.

The first phase uses the coordinates (latitude and longitude) of the trips per subject, and the TLG network in order to identify the trips crossing bridges, and the bridges crossed. The TLG network refers to a digital map maintained by the Metropolitan Council and The Lawrence Group (TLG). It covers the entire 7-county Minneapolis-St. Paul Metropolitan Area and is the most accurate GIS map of this network to date. The TLG network contains 290,231 links, and provides an accurate depiction of the entire Minneapolis-St. Paul network at the street level. The identification is done by spatial matching the coordinates of each bridge of interest to the coordinates of each set of trips for each subject. Also, subjects' trips must start at their home/work and end at their work/home locations in order to be considered commute trips (only direct commute trips). The distance tolerance between origins (destinations) to home (work) locations was set to 600 meters. The home and work locations are geocoded (transformed into latitude and longitude coordinates) from the actual addresses provided by the subjects on the web-based surveys. The origin and destination pair of each trip is obtained by mapping the coordinate points into trajectories of engine-on and engine-off events. Moreover, inaccurate points due to GPS "noise", and out-of-town trips (e.g. during Thanksgiving) were excluded. Also, only the trips after September 18th are considered as this is the date the new I-35W Bridge opened to the public at 5 AM. Lastly, only morning trips (those between 4 AM and 11 AM) are considered, because it is likely that subjects are not able to gather information from other non-commute trips during the morning, especially when the subjects drive directly from home to work without any side stops.

The second phase extracts usable information from the matched trips such as: statistics of travel time distribution of all trips (e.g. mean, standard deviation, and others) for each subject from GPS data. This process is performed for home to work trips. This is further explained in section 4.

The third phase is explained in section 4 . 

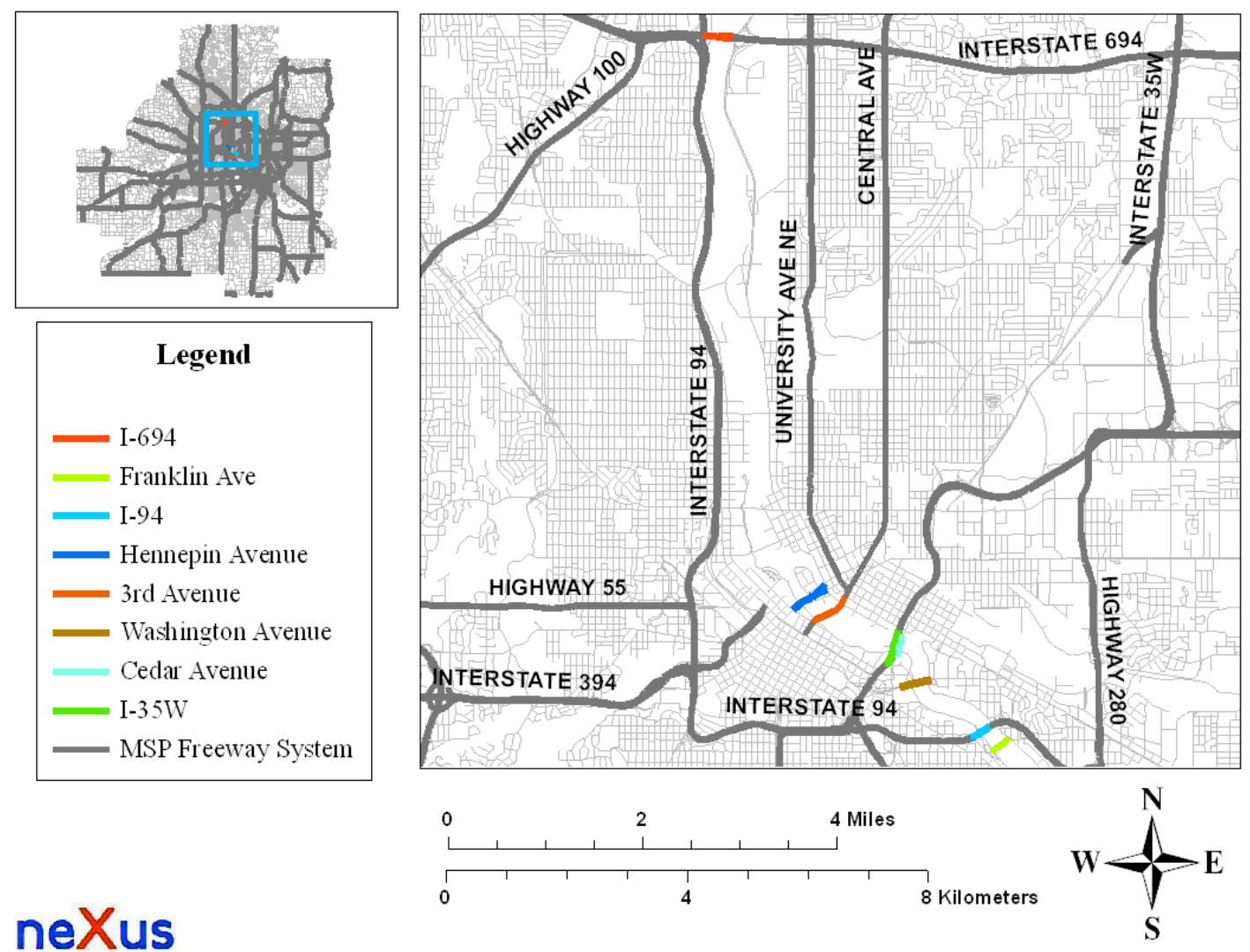

neXus

Figure 1: Bridge locations (Source: (1)) 


\section{3.3 Descriptive statistics}

3 Table 1, summarizes socio-demographic information of the subjects. The sample differs from the population

4 of the Minneapolis-St. Paul region in several ways: subjects are older, more educated, wealthier, and the 1 proportion of females is significantly greater than the proportion of males.

Table 1: Socio-Demographics attributes of the sample

\begin{tabular}{|l|l|l|l|}
\hline Number of Subjects & & 65 & \\
\hline & & Sample & Twin Cities \\
\hline Sex & Male & $27.69 \%$ & $49.40 \%$ \\
& Female & $72.31 \%$ & $50.60 \%$ \\
\hline Age (Mean, Std. Deviation) & & $(51.11,10.31)$ & $(34.47,20.9)$ \\
\hline Education & 11th grade or less & $0.00 \%$ & $9.40 \%$ \\
& High School & $15.87 \%$ & $49.60 \%$ \\
& Associate & $23.81 \%$ & $7.70 \%$ \\
& Bachelors & $46.03 \%$ & $23.20 \%$ \\
& Graduate or Professional & $14.29 \%$ & $10.10 \%$ \\
\hline Household Income & $\$ 49,999$ or less & $26.15 \%$ & $45.20 \%$ \\
& $\$ 50,000$ to $\$ 74,999$ & $20.00 \%$ & $23.30 \%$ \\
& $\$ 75,000$ to $\$ 99,999$ & $32.31 \%$ & $14.60 \%$ \\
& $\$ 100,000$ to $\$ 149,999$ & $18.46 \%$ & $11.00 \%$ \\
& $\$ 150,000$ or more & $3.08 \%$ & $5.90 \%$ \\
\hline Race & Black/African American & $9.52 \%$ & $6.20 \%$ \\
& White or Caucasian & $76.19 \%$ & $87.70 \%$ \\
& Others & $14.28 \%$ & $6.10 \%$ \\
\hline
\end{tabular}

Minneapolis' Population statistics are obtained from the (31) 


\section{Econometric models}

In this study, the data set is analyzed through duration analysis (also know as failure time analysis in operations research; hazard analysis in insurance and accident theory; and survival analysis in biostatistics) $(32,33,34)$. The dependent variable is the single-spell duration per subject. This duration is defined as the date and time elapsed from September 18th 5:00 AM until the date and time a subject consistently leaves his current bridge choice for any other of those in figure 1 (transition is observed), or the date and time until the GPS device is retrieved from the subject, and the subject has not left his current bridge choice (transition is not observed). The term current bridge choice refers to the subject's bridge choice at or after September 18th 5:00 AM. The term consistently refers to a subject's transition from his current bridge choice to another bridge at least two times consecutively. The term single-spell duration refers to modeling only one single transition from the current bridge choice to any other of those in figure 1. This single transition is only the first transition observed in the subjects' GPS data. In the data set, there are only 65 subjects, and only 65 single-spell transitions (one single-spell observation per subject). There are 835 observations, and on average 12.84 observations per subject. In addition, subjects are observed on average for 32.06 days, and 34 subjects are observed to consistently transition from their current bridge choice to another bridge of those in figure 1 . Also, the order of the events (i.e. different observed days and times of subjects' trips) of the single-spell duration per subject is known to the minute (year, month, day, hour, and minutes).

\subsection{Duration models}

\subsubsection{Cox Proportional Hazard model}

Duration models, similar to other econometric models, may have nonparametric, semi-parametric, and/or parametric models for the dependent variable. The Cox Proportional Hazard (hereafter referred as Cox $\mathrm{PH})$ model is a semi-parametric model that assumes the hazard function (conditional on the parameters and covariates; $h(t \mid \beta, x)$ ) is factored into two separate functions (proportional hazards assumption).

$$
h(t \mid \beta, x)=h_{0}(t) \phi(x, \beta)
$$

$h_{0}(t)$ is known as the baseline hazard, and $\phi(x, \beta)$ is known as the relative hazard. The baseline hazard is a function of the date, and time of transition, and its functional form is left unspecified. The shape of the baseline hazard function depends on the data, and it is not assumed as in fully parametric models. The baseline hazard function is estimated using a nonparametric product limit estimator similar to the Kaplan-Meier estimator $(32,34)$. This is further explained in section 4.1.4. The relative hazard is a nonnegative function (i.e. hazard rates cannot be negative) that is fully specified by the researcher. The common functional form of $\phi(x, \beta)$ is the exponential $\left(\phi(x, \beta)=e^{\beta^{T} x} ; \beta\right.$ is a vector of coefficients, and $x$ are the vectors of covariates in the regressors matrix). The relative hazard is estimated by maximizing a Partial Likelihood function. This is further explained in section 4.1.4. For this study, the hazard rate is

$$
h(t \mid \beta, x)=h_{0}(t) e^{\beta^{T} x}
$$

The basic assumption of the Cox PH's hazard function is that all subjects have the same baseline hazard function $\left(h_{0}(t)\right)$, and the relative hazard $(\phi(x, \beta))$ has a multiplicative effect on the baseline hazard depending on the values of the covariates. In other words, one subject's hazard function is a multiplicative version of another subject's hazard function. This is the proportional hazards assumption, and it is statistically tested for the data set of this study. This is further explained in section 4.1.3 along with other statistical tests. 
1 Furthermore, the Cox PH model may be adjusted to consider: tied observations; and censorship of single2 spell durations. The tied observations refer to the lack of information with regards to the order of the subjects that transition at the same date, and time in the data set. The order matters as it is required for the estimation of Cox PH model. In this study, there are no ties as the order of the events (i.e. different observed days and times of subjects' trips) of the single-spell duration per subject is known to the minute (year, month, day, hour, and minutes). The censorship (or more precisely right-censorship) of single-spell durations refer to the researchers not observing the transition of the subjects. An observed transition is defined as a subject consistently leaving his current bridge choice for any other of those in figure 1. A unobserved transition is a subject not leaving his current bridge choice before the GPS device is retrieved from the subject. In other words, the study ends before a transition is observed. Readers should remember that the term current bridge choice refers to the subject's bridge choice at or after September 18th 5:00 AM. In this study, there are 34 subjects (out of a total of 65 subjects) are observed to consistently transition (i.e. at least two times consecutively) from their current bridge choice to another bridge of those in figure 1. In contrast, there are 31 subjects with unobserved transition. It is assumed that the censorship mechanism is independent from the single-spell duration of the subjects. This is a fair assumption given that the time that a GPS device is retrieved from a subject did not depend on whether a subject changed bridge choices or not, but rather on the fixed time duration of the study (i.e. 8 or 13 weeks depending on the data collection effort; see section 3.1).

\subsubsection{Relative hazard: covariates}

The selection of the covariates in the relative hazard function is based on two major groups: characteristics of the subjects; and travel time measures. The characteristic of the subjects are obtained from web-based survey data, and GPS data. The travel time measures are calculated on travel times obtained from GPS data. Readers may refer to section 3.2 for details. In addition, there are two types of covariates: time-invariant $(y)$; and time-dependent $(z(t))$. Thus, the relative hazard is given by

$$
\phi\left(y, z(t) ; \beta_{x}, \beta_{z}\right)=e^{\beta_{y}^{T} y+\beta_{z}^{T} z(t)}
$$

$\beta_{y}$, and $\beta_{z}$ are vector of coefficients to be estimated. $y$ is a vector of time-invariant covariates (only change across subjects, and not across subjects' day-to-day morning commute trips). $z(t)$ is a vector of timedependent covariates (the values vary across subjects' day-to-day morning commute trips).

The time-invariant covariates $(x)$ are:

- $y_{1}$ : Past bridge diversity.

- $y_{2}$ : Gender.

- $y_{3}$ : Income.

- $y_{4}$ : Ratio of bridge distances (current bridge choice to previous bridge choice).

- $y_{5}$ : Fear of driving on the I-35W bridge and other bridges in the vicinity.

\section{Past bridge diversity $\left(y_{1}\right)$}

The number of distinct alternatives (bridges) a subject used for his morning commute trip before September 18th 5:00 AM. This covariate is an indication of a subject's knowledge of alternative bridges before traveling each day from September 18th 5:00 AM until the subject decided to change to another bridge alternative. 


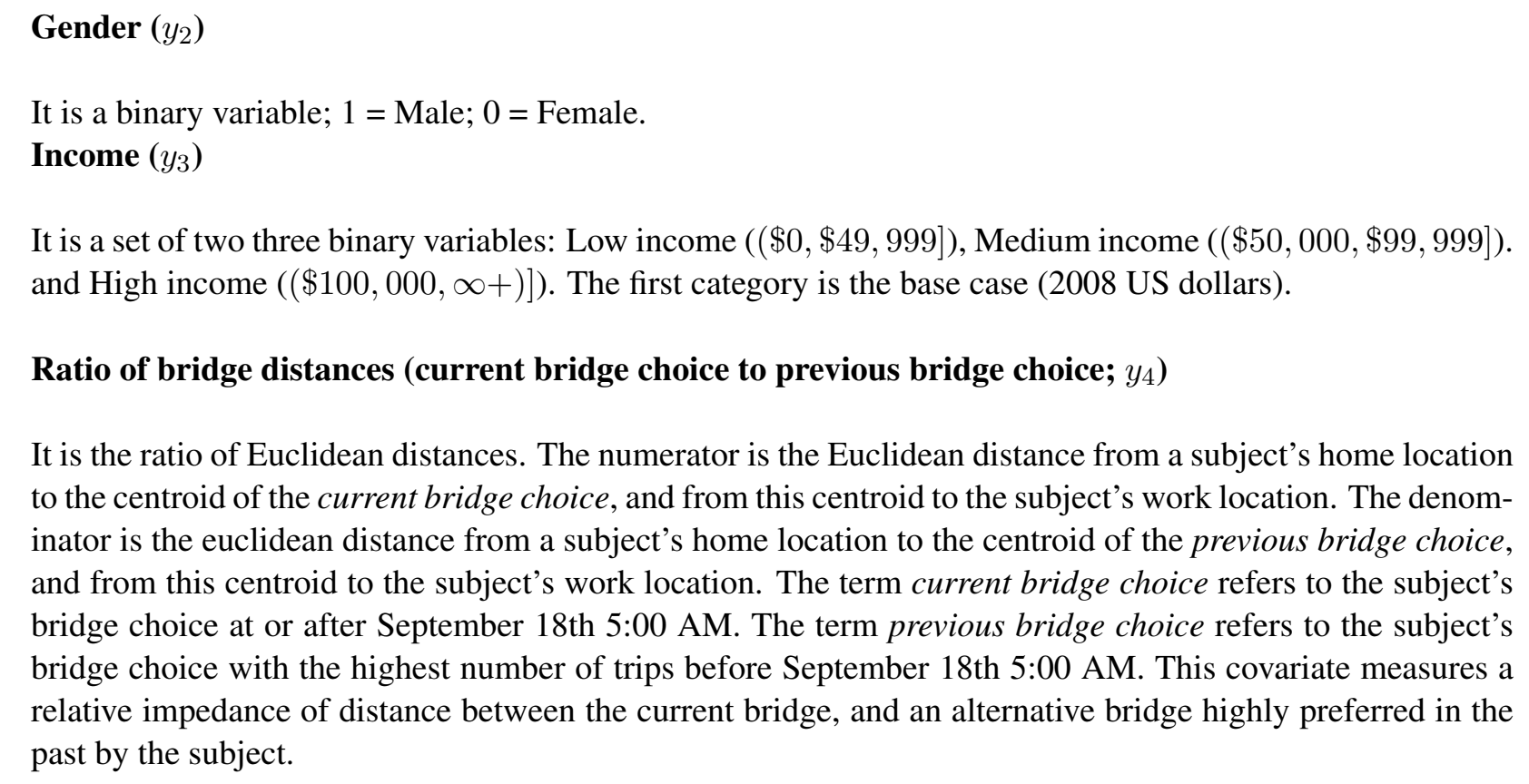

It is the ratio of Euclidean distances. The numerator is the Euclidean distance from a subject's home location to the centroid of the current bridge choice, and from this centroid to the subject's work location. The denominator is the euclidean distance from a subject's home location to the centroid of the previous bridge choice, and from this centroid to the subject's work location. The term current bridge choice refers to the subject's bridge choice at or after September 18th 5:00 AM. The term previous bridge choice refers to the subject's bridge choice with the highest number of trips before September 18th 5:00 AM. This covariate measures a relative impedance of distance between the current bridge, and an alternative bridge highly preferred in the past by the subject.

\section{Travel time measures}

In this study, the authors hypothesize that the subjects only consider a subset of their travel times, and that the subjects only recall every few days a portion of the travel times in this subset. In addition, the travel times in this subset are continuously updated according to thresholds set by the subjects. Figure 2a presents the day to day travel times of a subject in the sample. A (epanechnikov; 5.29 bandwidth) kernel weighted local polynomial smoother with its $95 \%$ confidence interval (see (32)) is fitted to the day to day travel times to further elucidate the trend across days. The smoother indicates that the travel times are constantly in flux until they finally increase significantly. Therefore, it must be asked how do travelers react to the trend and volatility of the observed travel time time series. It is reasonable given the perception of travelers that only certain travel times have weight in influencing the choices of travelers. In other words, travel times across days that are closely similar (e.g. 20 minutes, and 24 minutes) may not be as noticeable to the traveler in comparison to travel times across days that are quite dissimilar (e.g. 20 minutes and 35 minutes). Thus, 
it is assumed that the subjects consider traveler times above a certain number of standard deviations from the mean, and also travel times below a certain number of standard deviations from the mean. The standard deviation is calculated on the travel times of the travelers' trips, and the mean is calculated on the travel times of the travelers' trips. Two possible rules for thresholds are: fixed, and moving. The fixed thresholds (see figure 2b) assume travelers have an rigid expectation of the travel time, and a rigid magnitude for the travel time variability. Travel times within margins (e.g. mean plus one standard deviation, and mean minus one standard deviation) are acceptable and travel times above are not undesirable, and travel times below may also not be preferred. In addition, the margins may be asymmetric indicating that travelers may be more forgiving of travel times below the mean, but not so much of travel times above the mean. The moving thresholds (see figure 2c) assumes travelers' expectation of travel time, and travelers' variability of travel time are continuously updated according to the travelers' travel times of past trips. The mean is a moving mean that may only consider the travel time of two or more previous trips, and the standard deviation is a moving standard deviation that may also only consider the travel time of two or more previous trips. The shorter the number of previous trips considered the closer is the moving mean to the actual travel times. The margins are continuously updated, and thus travel times, that may not be acceptable (i.e. within the thresholds) in past trips, are acceptable in future trips. Furthermore, travelers, besides having fixed or moving thresholds, may also have a fixed or moving set of travel times that they can remember or recall. Travelers may discard (or forget) the travel times, and the frequency of past trips beyond their thresholds, and within their thresholds. This set of travel times is likely to be updated across days, and thus effectively travelers may only remember travel times and frequencies of past trips up to a specific number of days.

Lastly, the influence of the travelers' past trips on their previous most traveled bridge choice is considered. It is plausible that travelers at the beginning of their trips in their current bridge choice may contrast the current travel experience on the current bridge with the past travel experience on the past bridge. In addition, the travelers may eventually forget about their past travel experience on the past bridge. Also, it is plausible that travelers may change to other bridges, because they are familiar with these bridges without necessarily remembering their previous travel experience, or possessing a travel experience on these bridges. It is hypothesized that travelers compare the difference between the median of the travel times of their previous most traveled bridge choice with the each of the travel times experienced by the travelers for each trip.

The proposed travel time measures of this study are: fixed thresholds defined by mean, and number of standard deviations from the mean; moving thresholds defined by moving means, and a number of moving standard deviations from the moving mean; and travel times from the previous most used bridge.

\section{Fixed thresholds $\left(z_{1}(t)\right)$}

The mean, and the standard deviation of the travel times of a subject's set of morning commute trips is calculated. Furthermore, the authors test thresholds that are $0.5,1,2$, and 3 times from the mean. The authors test both symmetric thresholds (e.g. mean - 0.5 standard deviation, and mean +0.5 standard deviation), and asymmetric thresholds (e.g. mean - 0.5 standard deviation, and mean +1 standard deviation). In addition, a moving set of trips is considered. This moving set include trips that are from 2 to 15 days ago from the specific day of travel of a trip. In other words, the set considers exactly a fixed number of trips right before each of current trips a subject undertook. These variables measure the proportion of trips (number of trips divided by total number of trips) that are above (e.g. travel time greater than mean plus one standard deviation), and below (e.g. travel time less than mean plus one standard deviation) the thresholds. Proportion of trips within the thresholds are considered. The authors refer to trips above the thresholds as late trips, and to trips below the thresholds as early trips. Trips within the thresholds are regular trips. 


\section{Moving thresholds $\left(z_{2}(t)\right)$}

The moving mean, and the moving standard deviation of the travel times with distinct thresholds that are $0.5,1,2$, and 3 times from the moving mean are considered. The moving mean, and the moving standard deviation are calculated on a moving set that includes trips that are from 2 to 15 days ago from the specific day of travel of a trip. The moving set considers exactly a fixed number of trips right before each of current trips a subject undertook. These variables measure the proportion of trips (number of trips divided by total number of trips) that are above (e.g. travel time greater than moving mean plus one moving standard deviation), and below (e.g. travel time less than moving mean plus one moving standard deviation) the thresholds. Proportion of trips within the thresholds are considered. The authors refer to trips above the thresholds as late trips, and to trips below the thresholds as early trips. Trips within the thresholds are regular trips.

\section{Travel times from the previous most used bridge $\left(z_{3}(t)\right)$}

The bridge with the highest number of trips before September 18th 5:00 AM is identified. The median of the travel times of the different days a traveler used the bridge is calculated. The difference between this median, and each of the days travel times is computed. Furthermore, it is assumed that the coefficient of this time-dependent covariate is also time-dependent. Basically, the coefficient for the first week of travel of a subject in the current bridge choice is different from the coefficient after the first week of travel in the current bridge choice.

\subsubsection{Hypothesis testing and goodness of fit}

There are two hypothesis tests that are considered for the duration models in this study. For the nested models, the Wald tests are used as they only depend on the covariance matrix of the unrestricted models, and do not require estimation of the restricted models. These tests are asymptotically equivalent to the likelihood ratio tests. For the nonnested models, the Akaike information criterion (AIC), and Bayesian information criterion (BIC) are used in order to compare the statistical fit of the duration models with the different travel time measures proposed in section 4.1.2. See $(35,36,37)$ for more details.

The goodness of fit of the models are checked with residual analysis $(32)$. The Schoenfeld residuals $(38,39)$ are computed to test the proportional hazards assumption required for the Cox PH model, and the Deviance residuals (40) are examined to check the model accuracy, and identification of outliers.

\subsubsection{Estimation}

The estimation of the Cox PH model is done in two steps. The first step is the maximization of a Partial Likelihood function to obtain estimates of the coefficients, and the covariance matrix for the coefficients in the relative hazard function. The second is the maximization of a product limit estimator to obtain the hazard rate contributions given the estimates of the relative hazard. This estimator is a nonparametric Likelihood function. A thorough treatment is presented in $(32,34)$.

The Partial Likelihood function for this study is

$$
L\left(\beta_{y}, \beta_{z}\right)=\prod_{j=1}^{k} \frac{e^{\beta_{y}^{T} y_{j}+\beta_{z}^{T} z_{j}(t)}}{\sum_{m \in R\left(t_{j}\right)} e^{\beta_{y}^{T} y_{m}+\beta_{z}^{T} z_{m}(t)}}
$$

The $k$ is the number of single-spell durations, and thus of subjects in this sample. There is an order of these single-spell durations (i.e. $t_{1}<t_{2}<\ldots<t_{k}$ ). This order is present in the set $R\left(t_{j}\right)$. This is the set 
Figure 2: Thresholds-based behavior of travelers

(a) Day to day (GPS) travel times of a subject

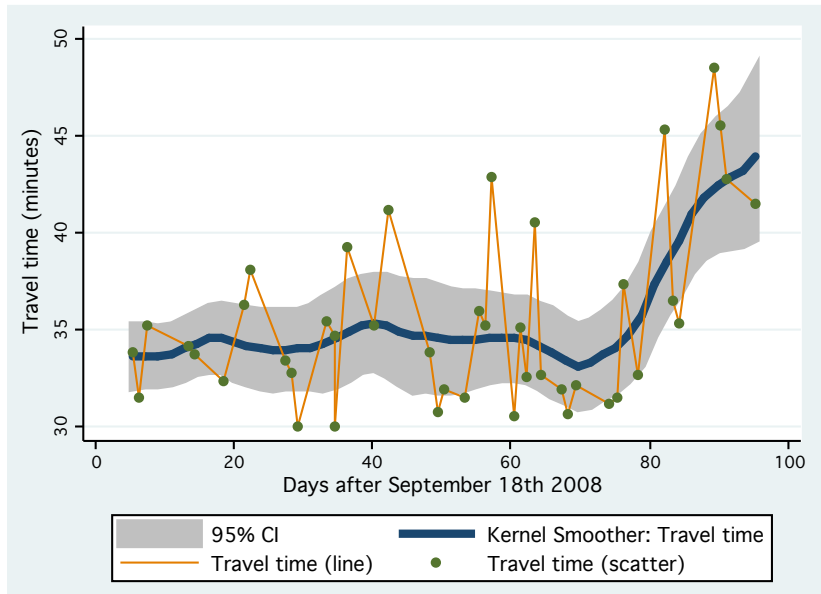

(b) Fixed thresholds

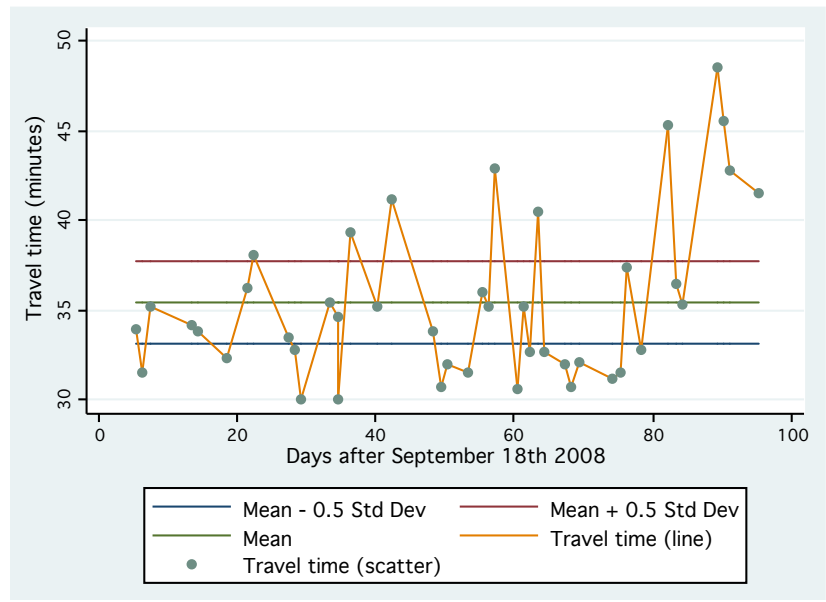

(c) Moving thresholds

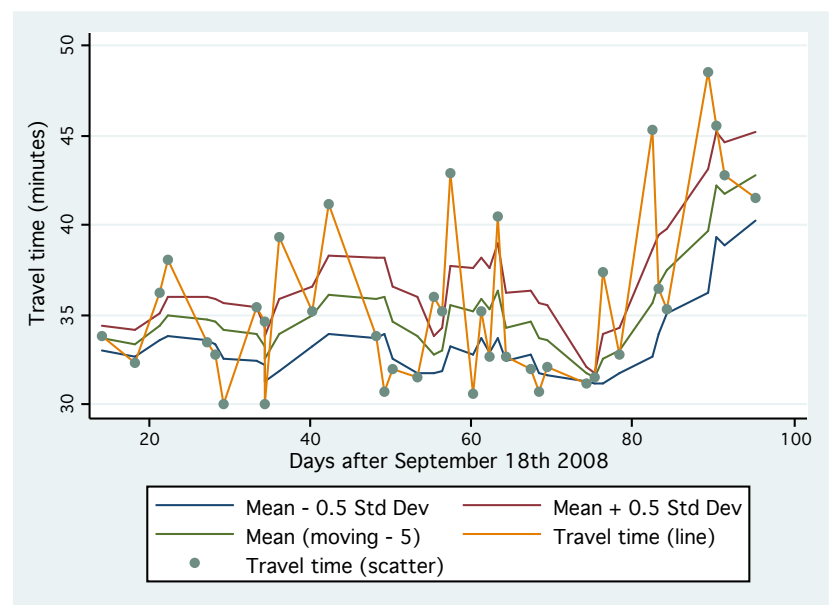


of single-spell durations that have transitioned at the order $j$, and that have not transitioned at the order $j$ excluding those that already transitioned before the order $j$. Mathematically, this is $R\left(t_{j}\right)=\left\{l: t_{l} \geq t_{j}\right\}$.

The nonparametric Likelihood function for this study is

$$
L\left(\alpha ; \hat{\beta}_{y}, \hat{\beta}_{z}\right)=\prod_{j=1}^{k}\left(\left(\alpha_{j}^{-e^{\hat{\beta}_{y}^{T} y_{j}+\hat{\beta}_{z}^{T} z_{j}(t)}}-1\right) \prod_{m \in R\left(t_{j}\right)} \alpha_{j}^{-\hat{\beta}^{T} y_{m}+\hat{\beta}_{z}^{T} z_{m}(t)}\right)
$$

The $1-\alpha_{j}$ are the baseline hazard rate contributions at the order $j$. This nonparametric Likelihood function allow the estimation of the Survivor function, and the contributions may be used to obtained a kernel smoothing function of the hazard rate function. This estimator is similar to the Kaplan-Meier estimator, but adjusted for the value of relative hazard's covariates.

The models are estimated using STATA (41). The plots are also obtained using STATA (42).

\section{Discussion and results}

Table 2 presents the estimates of the relative hazard functions of the models, and figures $4 \mathrm{a}, 4 \mathrm{~b}$, and $4 \mathrm{c}$ present the estimates of the baseline cumulative hazard, baseline hazard rate function, and baseline survivor function for the Fixed Thresholds 2 model. There are two types of models: Fixed Thresholds, and Moving Thresholds. The Fixed Thresholds models assume subjects have a rigid expectation with regards to their travel times, and also have a rigid magnitude of the travel time variability. Subjects classify their experience trips whether they fall into the margins (i.e. regular trip, fall above the margins (i.e. late trip), and below the margins (early trip). The Moving Thresholds models assume subjects continuously update their margins based on previous past trips. Similar to the Fixed Thresholds models, subjects classify their experience trips whether they fall into the margins (i.e. regular trip, fall above the margins (i.e. late trip), and below the margins (early trip). Readers should refer to see section 4.1.2 for details. In addition, both Fixed Thresholds and Moving Thresholds assume that subjects have a moving set of travel times. The moving set of travel times refer to which travel times of past trips the subjects are able to recall. Furthermore, readers should remember that the dependent variable of the model is the single-spell duration (as defined in section 4) of the subjects in their current bridge until they decide to switch to another bridge or until their GPS devices are collected from their vehicles.

For the models (Fixed Thresholds and Moving Thresholds), several combinations of distinct levels of standard deviations (or moving standard deviations) from the mean (or moving mean), and of past trips in the moving set of travel times were tested. The results that were statistically significant are summarized in table 2. All the models find that the number of past trips for classifying early trips are less than four past trips. In contrast, the number of past trips for classifying late trips is greater than 3 past trips, and most of the time its value was found to be 6 past trips. This indicates that subjects were found to recall further in time travel experiences of greater travel times with respect to the mean (or moving mean) in comparison to travel experiences of smaller travel times with respect to the mean (or moving mean). In addition, 0.5 standard deviation (or moving standard deviation) from the mean (or moving mean) were found to be statistically significant at least 5\% level for the margins classifying early trips. For the margins classifying late trips, 1 standard deviation (or moving standard deviations) from the mean (or moving mean) were found to be statistically significant at least 5\%. This implies subjects have asymmetric margins for classifying a trip to be late or early. Also, subjects consider trips not too far below the mean (or moving mean) as early, but trips farther above the mean are considered as late. Thus, subjects are tolerate travel times that are above, but close to the 
mean (or moving mean). Moreover, the signs indicate that the subjects are more likely to leave the current bridge choice if the number of late trips increases, and more likely to stay in the current bridge choice if the number of early trips increases. Readers should remember that Moving Thresholds models use moving means, and moving standard deviation, and the Fixed Threshold models use mean, and standard deviation. On the other hand, the addition of variables representing travel times of the subjects from past bridges were not found statistically significant. Thus, the question is whether subjects have a clean slate, or the inclusion of these variables requires a better hypothesis of how travelers see past time. It was previously discussed (see section 4.1.2 and section 2.1) that travelers may eventually forget about their past travel experience on other bridges. It is also plausible that travelers may change to other bridges, because they know of their existence, and not necessarily because they remember their previous travel experience. An interesting result of these variables, albeit not statistically significant, is that the sign is negative for the first week, and it is positive for the rest of the single-spell duration. This means that subjects with higher travel times on their past bridges are likely to stay in their current bridge choice during the first week. Similarly, traditional travel time measures from the Centrality-Dispersion framework, and the Scheduling under uncertainty framework were not found statistically significant. Thus, we must question whether these frameworks are only reflecting the aggregate responses of subjects (i.e. static assumption), and are not able to capture the dynamics across the responses of subjects (see section 2.1).

Other important results are that the previous knowledge (i.e. before September 18th 2008) of other bridges is statistically significant at $1 \%$. This previous knowledge (or past bridge diversity) has a positive sign indicating that subject familiar with other bridges are more susceptible to leave their current bridge choice. The ratio of bridge distances between the most used past bridge, and the current bridge choice indicates that subjects are influenced (statistically significant at $5 \%$ ) by travel distance. Subjects prefer bridges that are closer for their home to work trips. Subjects are more susceptible to leave the current bridge choice is they know of a previous past bridge that is closer to them. In addition, subjects that indicated in the surveys fear of bridges are more susceptible (statistically significant at least 10\%) to leave current bridge choice as long as the current bridge choice is the I-35W bridge or other bridges in the vicinity (i.e. Washington Ave bridge, and 10th St bridge). Lastly, subjects with income levels were found statistically significant at least 5\% (see section 4.1.2). The sign of these variables is negative, and thus indicating a reluctance to leave their current bridge choice. These variables require further research to identify the reason behind them. It is hypothesized that perhaps the type of jobs may play a role.

Finally, the goodness of fit analysis indicate that the Cox PH models' proportional hazard hypothesis cannot be rejected (i.e. Schoenfeld p-values), and the Deviance plots indicate for most models that there is no pattern, except a slight pattern for the Moving Thresholds models. Moreover, the Cox PH model preferred according to Akaike Information Criteria, and Bayesian Information criteria is the Fixed Thresholds 2 model. Figures $4 \mathrm{a}, 4 \mathrm{~b}$, and $4 \mathrm{c}$ present the estimates of the baseline cumulative hazard, baseline hazard rate function, and baseline survivor function for the Fixed Thresholds 2 model. The baseline cumulative hazard indicates that there is rapid growth in the susceptibility to leave the current bridge choice, and that eventually it flattens. This rapid growth happens before 40 days after the date September 18th 2008. This agrees with the baseline hazard rate function that most of the susceptibility to leave the current bridge choice occurs before 40 days after the date September 18th 2008. In addition, the susceptibility of leaving the current bridge choice increases until 20 days September 18th 2008, and eventually the susceptibility decreases. This agrees with the baseline survivor function that indicates a sharp drop in the survival probability by 20 days after September 18th 2008, and a smoother drop between 20 days after September 18th 2008, and 40 days after September 18th 2008. 


\section{Conclusion}

In this study, the authors have tried to uncover the dynamics behavior of subjects by using GPS data, and using an alternative modeling approach (duration models) to the Random Utility models. Cox PH models are fitted to the single-spell durations of travelers after a new bridge replacing the collapsed I-35W bridge in Minneapolis opened to the public. Several key difficulties are identified: travelers perceive the travel times at the end of each of their trips; , and travelers recall travel times from past trips. Thus, two important concerns are: the selection of day to day travel time by travelers due to their perception; and the ability of the travelers to recall the previously selected day to day travel times. It should be noted that these concerns are similar to those put forward in a more general setting by (30) (i.e. bounded rationality). Furthermore, two types of models are proposed: Fixed Thresholds models, and Moving Thresholds models. The Fixed Thresholds models assume subjects have a rigid expectation with regards to their travel times, and also have a rigid magnitude of the travel time variability. Subjects classify their experience trips whether they fall into the margins (i.e. regular trip, fall above the margins (i.e. late trip), and below the margins (early trip). The Moving Thresholds models assume subjects continuously update their margins based on previous past trips. Similar to the Fixed Thresholds models, subjects classify their experience trips whether they fall into the margins (i.e. regular trip, fall above the margins (i.e. late trip), and below the margins (early trip). Readers should refer to see section 4.1.2 for details. In addition, both Fixed Thresholds and Moving Thresholds assume that subjects have a moving set of travel times. The moving set of travel times refer to which travel times of past trips the subjects are able to recall. Furthermore, readers should remember that the dependent variable of the model is the single-spell duration (as defined in section 4) of the subjects in their current bridge until they decide to switch to another bridge or until their GPS devices are collected from their vehicles.

The primary results indicate that both the Fixed Thresholds, and the Moving Thresholds models are found to capture the dynamics of the data, but the the Fixed Thresholds should be preferred. In addition, the late trips are more persistent in the subjects' travel time moving sets in comparison to the subjects' early trips. It is also found that both margins to classify whether a trip is an early trip or a late trip are asymmetric. Subjects are tolerant to small increases above the mean in their travel experiences. The secondary results indicate that subjects' perception (i.e. fear, travel distance) of the alternatives also influence their decision to abandon the chosen route. 


\section{References}

[1] C. Carrion and D. Levinson. A model of bridge choice across the mississippi river in minneapolis. In D. Levinson, H. Liu, and M. Bell, editors, Network Reliability in Practice: Selected papers from the fourth international symposium on transportation network reliability, chapter 8, pages 115-129. Springer, 2012.

[2] R.G. Golledge and R.J. Stimson. Spatial behavior. Guilford Press, New York, 1997.

[3] R. Golledge. Human wayfinding and cognitive maps. In Wayfinding Behavior: Cognitive Mapping and Other Spatial Processes, pages 5-45. John Hopkins, 1999.

[4] R. Golledge. Place recognition and wayfinding: Making sense of space. Geoforum, 23:199-214, 1992.

[5] P. Bovy and E. Stern. Route Choice: Wayfinding in Transport Networks. Kluwer Academic Publishers, Netherlands, 1990.

[6] M. Ben-Akiva, MJ Bergman, AJ Daly, and R. Ramaswamy. Modelling inter urban route choice behaviour. In Proceedings of the Ninth International Symposium on Transportation and Traffic Theory, Delft, the Netherland, pages 299-330, 1984.

[7] D. L. Trueblood. Effect of travel time and distance on freeway usage. Highway Research Board, (61): 18-37, 1952.

[8] R. D. Michaels. Attitudes of drivers toward alternative highways and their relation to route choice. Highway Research Record, (122):50-74, 1966.

[9] K. J. Kansky. Travel patterns of urban residents. Transportation Science, 1(4):261-285, 1967.

[10] L. E. Haefner and L. V. Dickinson. Preliminary analysis of disaggregate modeling in route choice. Transportation Research Record, (527):66-72, 1974.

[11] R. Hamerslag. Investigation into factors affecting the route choice in rijnstreek-west with the aid of a disaggregate logit model. Transportation, 10(4):373-391, 1981.

[12] M. Vaziri and T. N. Lam. Perceived factors affecting driver route decisions. Journal of Transportation Engineering, 109(2):297311, 1983.

[13] N. Tilahun and D. Levinson. A moment of time: Reliability in route choice using stated preference. Journal of Intelligent Transportation Systems, 14(3):179 -187, 2010.

[14] K.A. Small, C. Winston, and J. Yan. Uncovering the distribution of motorists' preferences for travel time and reliability. Econometrica, 73(4):1367-1382, 2005.

[15] K.A. Small, C. Winston, and J. Yan. Differentiated road pricing, express lanes, and carpools: Exploiting heterogeneous preferences in policy design. Brookings-Wharton Papers on Urban Affairs, 7:53-96, 2006.

[16] C. Carrion and D. Levinson. Value of reliability: High occupancy toll lanes, general purpose lanes, and arterials. In Conference Proceedings of 4th International Symposium on Tranportation Network Reliability in Minneapolis, MN (USA), 2010.

[17] L. Zhang and D. Levinson. Determinants of route choice and the value of traveler information: A field experiment. Transportation Research Record: Journal of the Transportation Research Board, 2086: 81-92, 2008. 
2 [18] M.A. Abdel-Aty, R. Kitamura, and P.P. Jovanis. Using stated preference data for studying the effect of advanced traffic information on drivers' route choice. Transportation Research Part C, 5(1):39-50, 1997.

[19] A. Pal. Modeling of commuter's route choice behavior. Master's thesis, The University of Toledo (USA), 2004.

[20] HK Wong and JM Sussman. Dynamic travel time estimation on highway networks. Transportation Research, 7:355-370, 1973.

[21] A. Nicholson and ZP Du. Degradable transportation systems: an integrated equilibrium model. Transportation Research Part B, 31(3):209-223, 1997.

[22] K. Train. Discrete choice methods with simulation. Cambridge University Press, 2nd edition, 2009.

[23] J. Ortuzar and L. Willumsen. Modelling Transport. Wiley, 4th edition, 2011.

[24] M. Ben-Akiva and S. Lerman. Discrete choice analysis: theory and application to travel demand. MIT Press, 1985.

[25] W. Jackson and J Jucker. An empirical study of travel time variability and travel choice behavior. Transportation Science, 16(4):460-475, 1982.

[26] K.A. Small. The scheduling of consumer activities: Work trips. American Economic Review, 72(3): 467-479, 1982.

[27] RB Noland and KA Small. Travel-time uncertainty, departure time choice, and the cost of morning commutes. Transportation Research Record, 1493:150-158, 1995.

[28] C. Carrion and D. Levinson. Value of reliability: A review of the current evidence. Transportation Research Part A, 46(4):720-741, 2012.

[29] T. Domencich and D. McFadden. Urban Travel Demand-A Behavioral Analysis. North Holland., 1975.

[30] H. Simon. Models of bounded rationality: empirically grounded economic reason, volume 3. MIT Press, 1997.

[31] 2006-2008 american community survey 3-year estimates, minneapolis-st. paul-bloomington, mn-wi metropolitan statistical area, retrieved november 25, 2009. URL http:// factfinder.census . gov/.

[32] A. C. Cameron and P. K. Trivedi. Microeconometrics: Methods and Applications. Cambridge Univ. Press, 2005.

[33] J. Wooldridge. Econometric Analysis of Cross Section and Panel Data. MIT Press, 2nd edition, 2010.

[34] J. Kalbfleisch and R. Prentice. The statistical analysis of failure time data. Wiley, 2nd edition, 2002.

[35] J. Johnston and J. DiNardo. Econometric methods. McGraw-Hill, 1997.

[36] W. Greene. Econometric Analysis. Prentice-Hall, 7th edition, 2012.

[37] J. Cramer. Econometric applications of Maximum Likelihood methods. Cambridge Univ. Press, 1986. 
2 [38] P. Grambsch and T. Therneau. Proportional hazards tests and diagnostics based on weighted residuals.

4

5

6 Biometrika, 81:515-526, 1994.

[39] D. Schoenfeld. Partial residuals for the proportional hazards regression model. Biometrika, 69:239$241,1982$.

[40] D. Collett. Modelling Survival Data in Medical Research. Chapman and Hall, 2nd edition, 2003.

[41] M. Cleves, R. Gutierrez, W. Gould, and Y. Marchenko. An Introduction to Survival Analysis using STATA. Stata Press, 3rd edition, 2010.

[42] M. Mitchell. A Visual Guide to STATA Graphics. Stata Press, 2nd edition, 2008. 


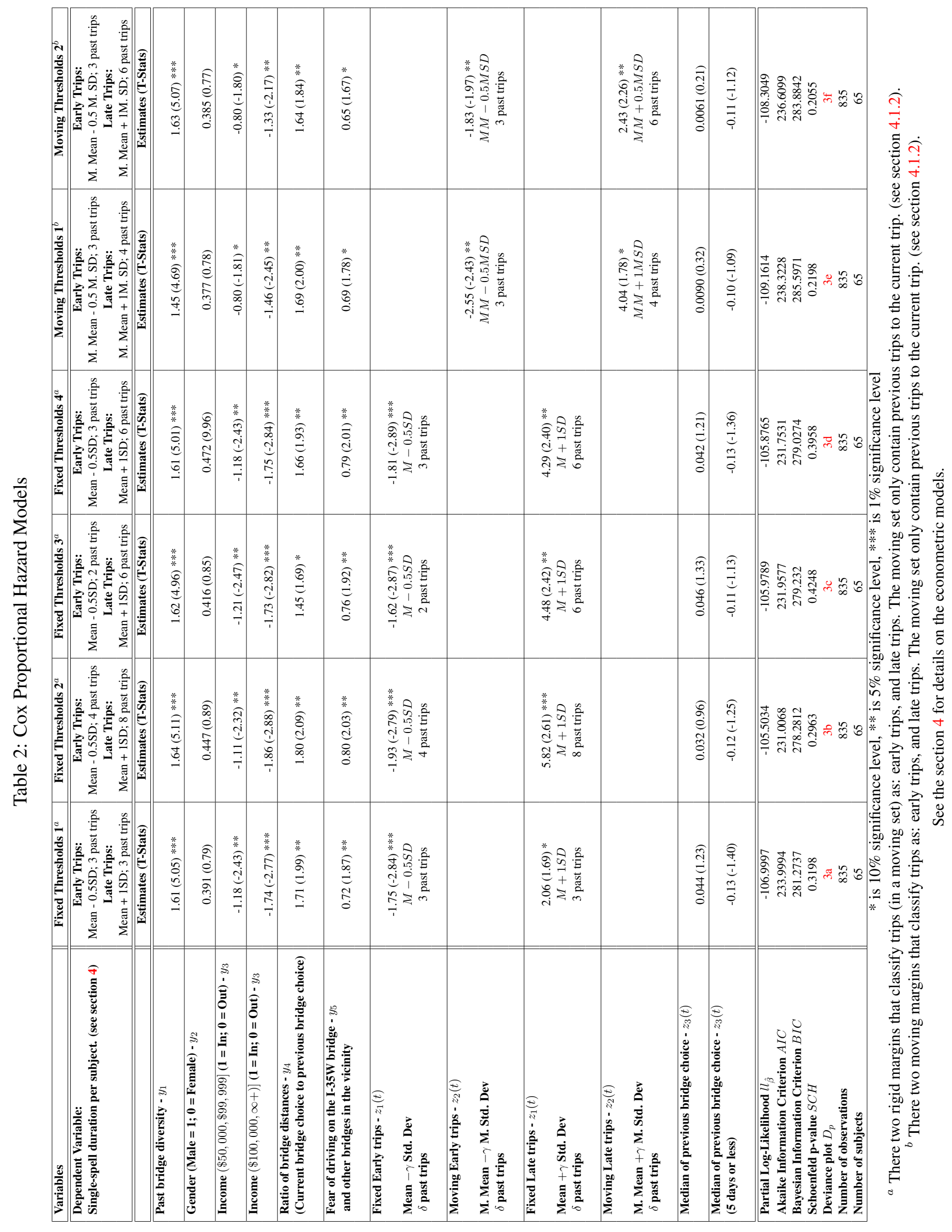


Figure 3: Deviance residuals plots

(a) Deviance Plot - Fixed Thresholds 1

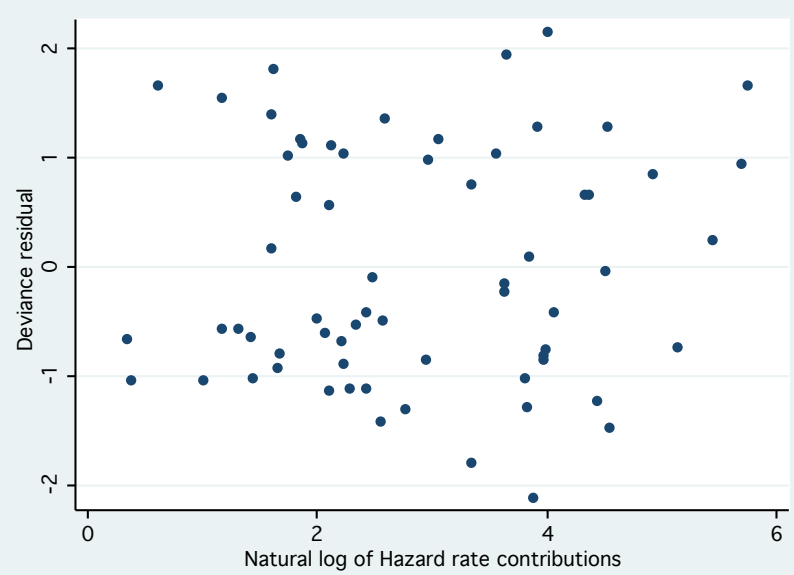

(c) Deviance Plot - Fixed Thresholds 3

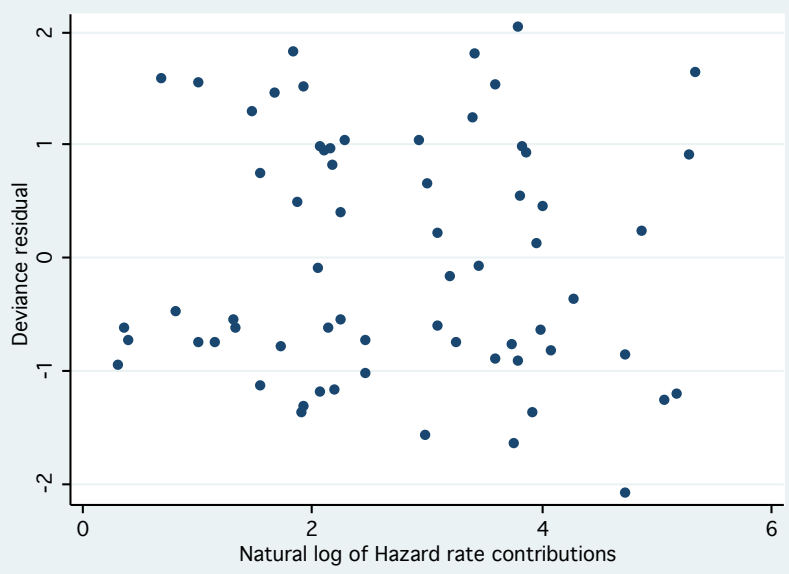

(e) Deviance Plot - Moving Thresholds 1

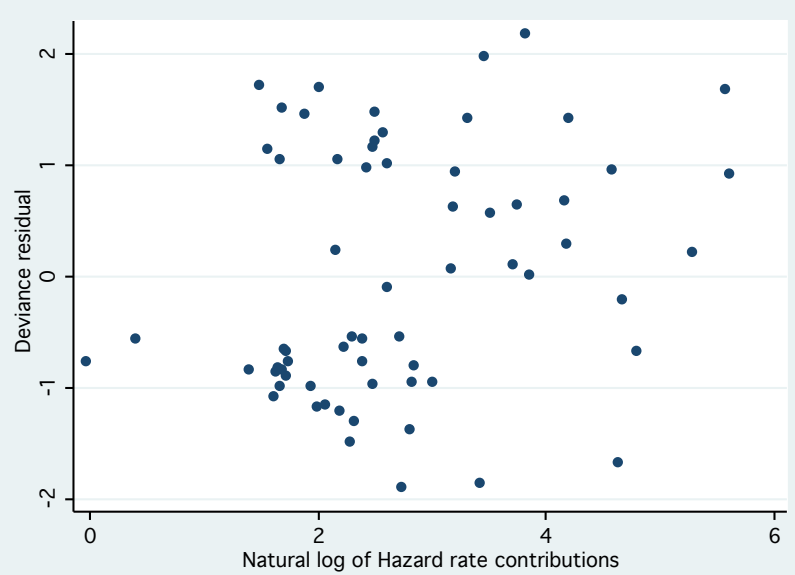

(b) Deviance Plot - Fixed Thresholds 2

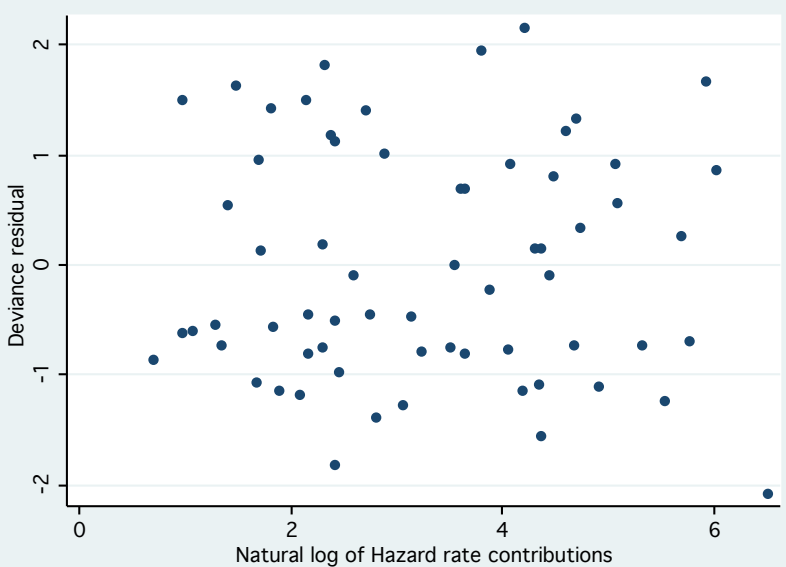

(d) Deviance Plot - Fixed Thresholds 4

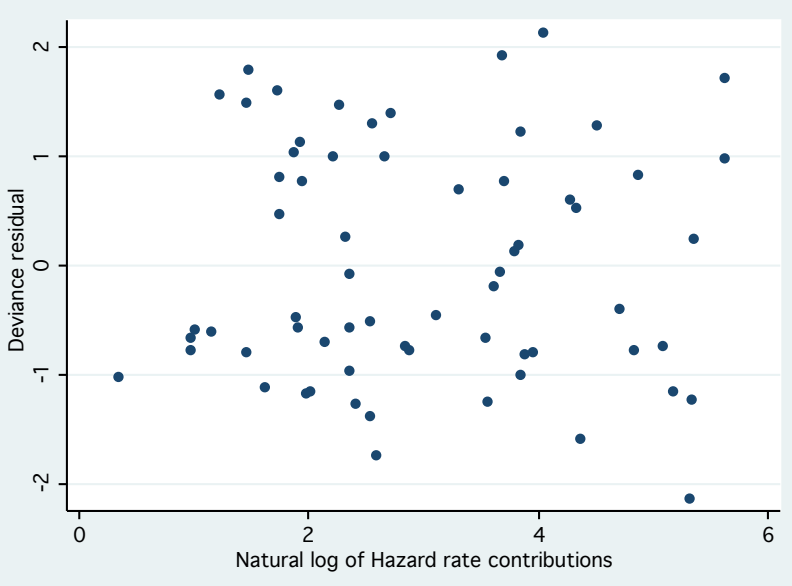

(f) Deviance Plot - Moving Thresholds 2

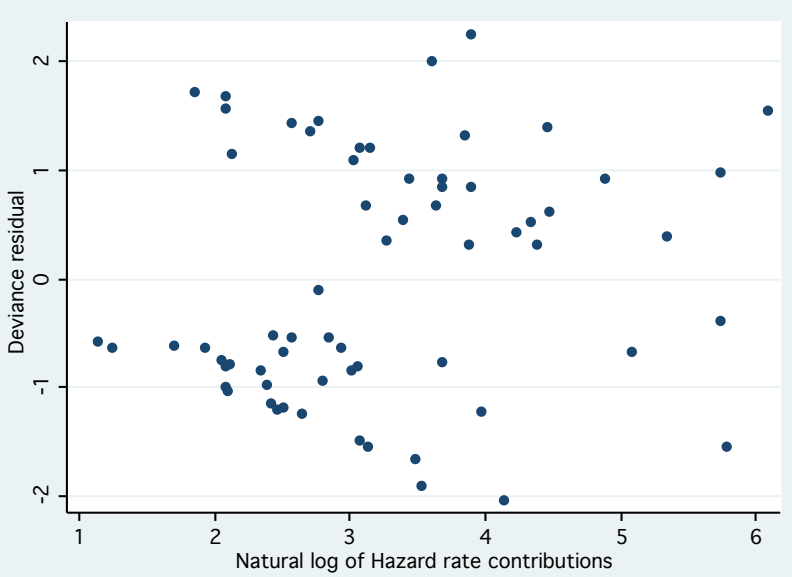


Figure 4: Baseline functions

(a) Baseline cumulative hazard - Fixed Thresholds 2

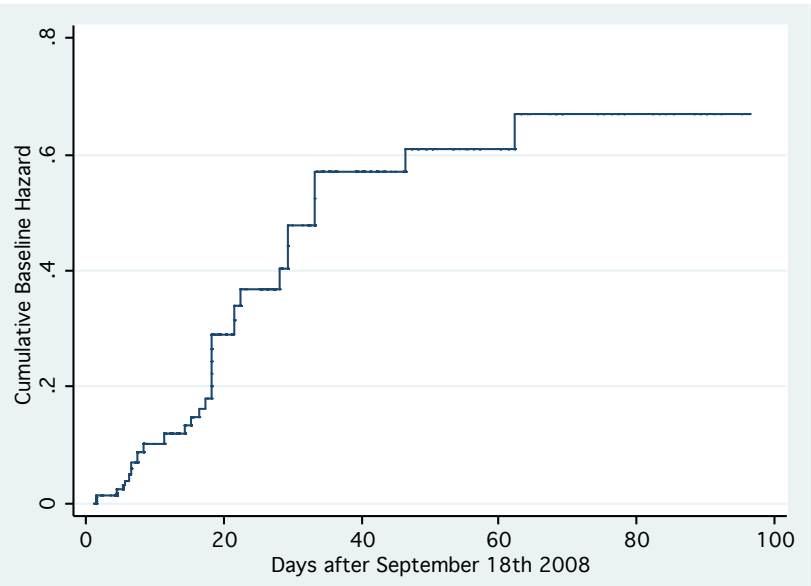

(c) Baseline survivor function - Fixed Thresholds 2

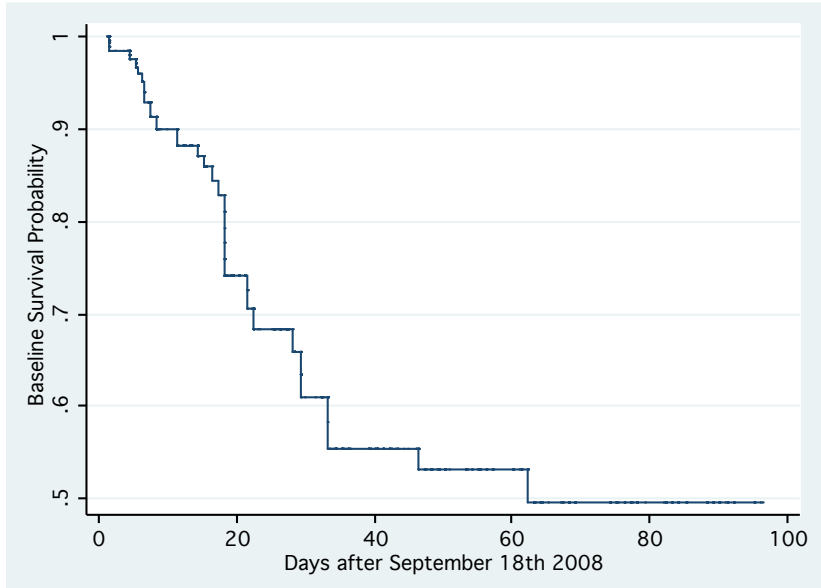

(b) Smoothed baseline hazard function - Fixed Thresholds 2

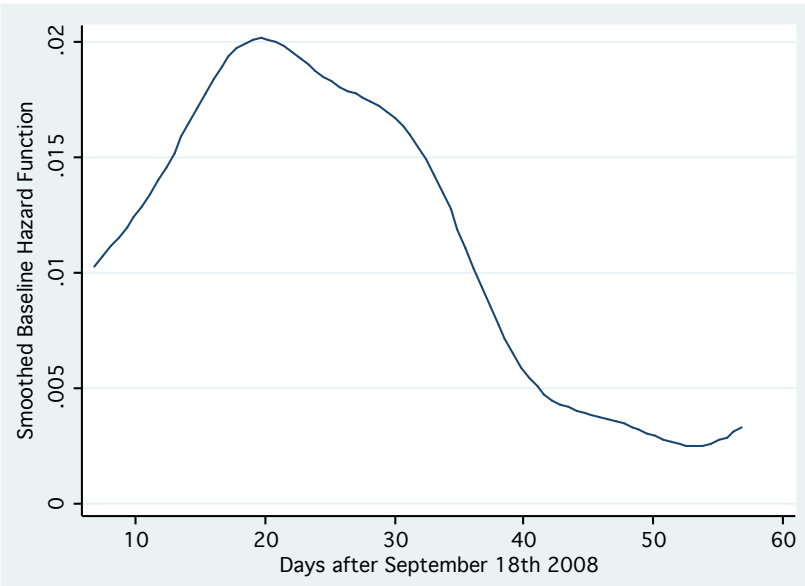

